\title{
Risk for Stillbirth Among Women With and Without COVID-19 at Delivery Hospitalization — United States, March 2020-September 2021
}

\author{
Carla L. DeSisto, PhD1; Bailey Wallace, MPH${ }^{1}$; Regina M. Simeone, $\mathrm{PhD}^{1}$; Kara Polen, MPH${ }^{1}$; Jean Y. Ko, $\mathrm{PhD}^{1}$;
} Dana Meaney-Delman, MD ${ }^{1}$; Sascha R. Ellington, $\mathrm{PhD}^{1}$

\section{On November 19, 2021, this report was posted as an MMWR} Early Release on the MMWR website (https://www.cdc.gov/mmwr).

Pregnant women are at increased risk for severe COVID-19related illness, and COVID-19 is associated with an increased risk for adverse pregnancy outcomes and maternal and neonatal complications (1-3). To date, studies assessing whether COVID-19 during pregnancy is associated with increased risk for stillbirth have yielded mixed results $(2-4)$. Since the B.1.617.2 (Delta) variant of SARS-CoV-2 (the virus that causes COVID-19) became the predominant circulating variant, ${ }^{*}$ there have been anecdotal reports of increasing rates of stillbirths in women with COVID- $19 .^{\dagger}$ CDC used the Premier Healthcare Database Special COVID-19 Release (PHD-SR), a large hospital-based administrative database, ${ }^{\S}$ to assess whether a maternal COVID-19 diagnosis documented at delivery hospitalization was associated with stillbirth during March 2020-September 2021 as well as before and during the period of Delta variant predominance in the United States (March 2020-June 2021 and July-September 2021, respectively). Among 1,249,634 deliveries during March 2020 September 2021, stillbirths were rare (8,154;0.65\%): 273 (1.26\%) occurred among 21,653 deliveries to women with COVID-19 documented at the delivery hospitalization, and $7,881(0.64 \%)$ occurred among 1,227,981 deliveries without COVID-19. The adjusted risk for stillbirth was higher in deliveries with COVID-19 compared with deliveries without COVID-19 during March 2020-September 2021 (adjusted relative risk $[\mathrm{aRR}]=1.90 ; 95 \% \mathrm{CI}=1.69-2.15)$, including during the pre-Delta $(\mathrm{aRR}=1.47 ; 95 \% \mathrm{CI}=1.27-1.71)$ and Delta periods $(\mathrm{aRR}=4.04 ; 95 \% \mathrm{CI}=3.28-4.97)$. COVID-19 documented at delivery was associated with increased risk for stillbirth, with a stronger association during the period of

\footnotetext{
* https://covid.cdc.gov/covid-data-tracker/\#datatracker-home

$\dagger$ https://msdh.ms.gov/msdhsite/_static/23,23645,341.html

$\$$ PHD-SR, formerly known as the PHD COVID-19 Database, is a large U.S. hospital-based, service-level, all-payor database that includes inpatient and hospital-based outpatient (e.g., emergency department or clinic) health care encounters from $>900$ geographically diverse nonprofit, nongovernmental, community, and teaching hospitals and health systems from rural and urban areas. PHD-SR represents approximately $20 \%$ of inpatient admissions in the United States. Data for this study represent a subset of 736 hospitals with delivery hospitalizations that contributed inpatient encounters to the PHD-SR during March 2020-September 2021. Updated PHD-SR data are released every 2 weeks; release date November 9, 2021, access date November 12, 2021. https://offers.premierinc.com/rs/381-NBB-525/images/PHD_COVID-19_ White_Paper.pdf
}

Delta variant predominance. Implementing evidence-based COVID-19 prevention strategies, including vaccination before or during pregnancy, is critical to reducing the impact of COVID-19 on stillbirths.

Delivery hospitalizations were identified from PHD-SR using International Classification of Diseases, Tenth Revision, Clinical Modification (ICD-10-CM) diagnostic and procedure codes pertaining to obstetric delivery and diagnosis-related group delivery codes. 9 Deliveries with discharge dates during March 2020-September 2021 were included. Stillbirths, defined as fetal deaths at $\geq 20$ weeks' gestation, were identified using maternal ICD-10-CM diagnosis codes. ${ }^{* *}$ Hospitalizations without ICD-10-CM codes indicating gestational age or with ICD-10-CM codes indicating gestational age $<20$ weeks were excluded to reduce misclassification of fetal deaths at $<20$ weeks' gestation as stillbirths (1.5\% of the overall sample).

Maternal demographic variables assessed included age, race/ ethnicity (i.e., Hispanic, non-Hispanic Black, non-Hispanic White, non-Hispanic Asian, and non-Hispanic other), and primary payor (i.e., Medicaid, private insurance, self-pay, and other). Assessed hospital characteristics included urban or rural location and U.S. Census division. COVID- $19^{\dagger \dagger}$ and selected underlying medical conditions (i.e., obesity, smoking, ${ }^{\$ \$}$ any diabetes, 99 any hypertension, ${ }^{* * *}$ and multiple-gestation

\footnotetext{
9 ICD-10-CM diagnostic and procedure codes pertaining to obstetric delivery: Z37.0, Z37.1, Z37.2, Z37.3, Z37.4, Z37.50, Z37.51, Z37.52, Z37.53, Z37.54, Z37.59, Z37.60, Z37.61, Z37.62, Z37.63, Z37.64, Z37.69, Z37.7, Z37.9, O75.82, O80, O82, 10D00Z0, 10D00Z1, 10D00Z2, 10D07Z3, 10D07Z4, 10D07Z5, 10D07Z6, 10D07Z7, 10D07Z8, 10E0XZZ; Diagnosis-related group delivery codes: $765,766,767,768,774,775,783,784,785,786,787$, 788, 796, 797, 798, 805, 806, 807; Excluded codes for ectopic or molar pregnancies and pregnancies with abortive outcomes: $\mathrm{O} 00, \mathrm{O} 01, \mathrm{O} 02, \mathrm{O} 03$, O04, O07, O08, Z33.2, 10A0. Deliveries with the O82 code were excluded if they did not cooccur with another delivery code. Females aged $12-55$ years were included. Multiple delivery events per woman during March 2020-September 2021 were included if the deliveries were $>6$ months apart.

** ICD-10-CM maternal diagnostic codes indicating a stillbirth: Z37.1, Z37.3, Z37.4, Z37.60, Z37.61, Z37.62, Z37.63, Z37.64, Z37.69, Z37.7. In multiplegestation pregnancies, if a woman experienced multiple stillbirths, she was counted once as experiencing a stillbirth. If she experienced both a live birth and a stillbirth during one delivery hospitalization, she was also counted once as experiencing a stillbirth.

†† COVID-19 was identified using ICD-10-CM code U07.1 (COVID-19, virus identified) during April 2020-September 2021 or B97.29 (Other coronavirus as the cause of disease classified elsewhere) during March-April 2020.

$\$ \$$ Includes smoking (tobacco) complicating pregnancy, childbirth, or the puerperium.

99 Includes prepregnancy diabetes and gestational diabetes.

*** Includes chronic hypertension; gestational hypertension; chronic hypertension with superimposed preeclampsia; preeclampsia; hemolysis, elevated liver enzymes, low platelet count (HELLP) syndrome; and eclampsia
} 
pregnancy) were included if the relevant ICD-10-CM diagnosis code was documented during the delivery hospitalization (3). In addition, among deliveries with documented COVID-19, indicators of severe illness (i.e., adverse cardiac event/outcome, ${ }^{\dagger \dagger \dagger}$ placental abruption, sepsis, shock, acute respiratory distress syndrome, mechanical ventilation, and intensive care unit [ICU] admission) were considered present if the relevant ICD-10-CM diagnosis code was documented during the delivery hospitalization (3). Vaccination status was unable to be assessed in this analysis.

Poisson regression models with robust standard errors were used to calculate overall unadjusted and adjusted ${ }^{\$ \$ \S}$ relative risks for stillbirth among deliveries with COVID-19 versus deliveries without COVID-19, accounting for within-hospital and withinwoman correlation. To better understand the potential biologic mechanism for stillbirth among women with COVID-19 at delivery, Poisson regression models with robust SEs were used to calculate unadjusted and adjusted $\boldsymbol{\Phi 9}$ prevalence ratios for stillbirth for each underlying medical condition and indicator of severe illness among deliveries with documented COVID-19. Relative risks and prevalence ratios were calculated overall as well as during the pre-Delta and Delta periods. Effect modification by period was assessed using adjusted models with interaction terms. For all models, p-values $<0.05$ were considered statistically significant. All analyses were performed using SAS software (version 9.4; SAS Institute). This activity was reviewed by CDC and was conducted consistent with applicable federal law and CDC policy.****

Among 1,249,634 deliveries at 736 hospitals during March 2020-September 2021, 53.7\% of women were nonHispanic White, and $50.6 \%$ had private insurance as the primary payor (Table 1). Overall, $15.4 \%$ had obesity, $11.2 \%$ had diabetes, $17.2 \%$ had a hypertensive disorder, $1.8 \%$ had a multiple-gestation pregnancy, and $4.9 \%$ had smoking (tobacco) documented on the delivery hospitalization record. Overall, 21,653 (1.73\%) delivery hospitalizations had COVID-19 documented.

${ }^{\dagger \dagger \dagger}$ Includes acute myocardial infarction, cardiomyopathy, heart failure/arrest during surgery or procedure, cardiac arrest/ventricular fibrillation, conversion of cardiac rhythm, incident ventricular tachycardia, ischemia, pulmonary edema/acute heart failure, and atrial fibrillation/atrial flutter/ supraventricular tachycardia.

$\$ \$ \$$ Models accounted for within-facility and within-woman correlation, and were adjusted for maternal age, race/ethnicity (Hispanic, non-Hispanic Black, non-Hispanic White, and non-Hispanic other), primary payor (Medicaid, private insurance, and other), obesity, smoking, any diabetes, any hypertension, and multiple-gestation pregnancy.

999 Models accounted for within-facility and within-woman correlation, and were adjusted for maternal age, race/ethnicity (Hispanic, non-Hispanic Black, non-Hispanic White, and non-Hispanic other), and primary payor (Medicaid, private insurance, and other).

**** 45 C.F.R. part 46, 21 C.F.R. part 56; 42 U.S.C. Sect. 241(d); 5 U.S.C. Sect. 552a; 44 U.S.C. Sect. Sect. 3501 et seq.
During March 2020-September 2021, a total of 8,154 stillbirths were documented, affecting $0.64 \%$ and $1.26 \%$ of deliveries without COVID-19 and with COVID-19, respectively $(\mathrm{aRR}=1.90 ; 95 \% \mathrm{CI}=1.69-2.15)$ (Figure). During the pre-Delta period (March 2020-June 2021), 6,983 stillbirths were documented, involving $0.98 \%$ of deliveries with COVID-19 compared with $0.64 \%$ of deliveries without COVID-19 $(\mathrm{aRR}=1.47 ; 95 \% \mathrm{CI}=1.27-1.71)$. During the Delta period (July-September 2021), 1,171 stillbirths were documented, involving $2.70 \%$ of deliveries with COVID-19 compared with $0.63 \%$ of deliveries without COVID-19 $(\mathrm{aRR}=4.04 ; 95 \% \mathrm{CI}=3.28-4.97) .{ }^{\dagger \dagger \dagger \dagger}$ Effect modification was present in the model; the risk for stillbirth was significantly higher during the period of Delta predominance than during the pre-Delta period $(\mathrm{p}<0.001)$.

Among deliveries with COVID-19, chronic hypertension, multiple-gestation pregnancy, adverse cardiac event/outcome, placental abruption, sepsis, shock, acute respiratory distress syndrome, mechanical ventilation, and ICU admission were associated with a higher prevalence of stillbirth (Table 2). The associations for adverse cardiac event/outcome and ICU admission varied significantly between the periods before and during Delta predominance $(\mathrm{p}=0.03$ and $\mathrm{p}=0.003$, respectively); for each of these, the associations were stronger during the period of Delta predominance.

\section{Discussion}

Although stillbirth was a rare outcome overall, a COVID-19 diagnosis documented during the delivery hospitalization was associated with an increased risk for stillbirth in the United States, with a stronger association during the period of Delta variant predominance. A previous study of pregnancies complicated by SARS-CoV-2 infection identified placental histopathologic abnormalities, suggesting that placental hypoperfusion and inflammation might occur with maternal COVID-19 infection (5); these findings might, in part, explain the association between COVID-19 and stillbirth. Among deliveries with COVID-19 documented during the delivery hospitalization, certain underlying medical conditions and markers of maternal morbidity, including the need for intensive care, were associated with stillbirth. Additional studies are warranted to investigate the role of maternal complications from COVID-19 on the risk for stillbirth. Further, given the differences observed before and during the period of Delta variant predominance, comparisons of placental findings might improve understanding of biologic reasons for the observed differences.

\footnotetext{
††† Sensitivity analyses were conducted to check for possible seasonality of stillbirths. In models using calendar year quarters, traditional seasons based on temperature patterns, and waves of SARS-CoV-2 variants, the results did not substantively change.
} 
TABLE 1. Maternal demographic and health characteristics and hospital characteristics among delivery hospitalizations with and without a documented COVID-19 diagnosis - Premier Healthcare Database Special COVID-19 Release, United States, March 2020-September 2021

\begin{tabular}{|c|c|c|c|c|c|c|c|}
\hline \multirow[b]{3}{*}{ Characteristic } & \multicolumn{7}{|c|}{ No. (\%) } \\
\hline & \multicolumn{3}{|c|}{$\begin{array}{c}\text { Overall } \\
N=1,249,634 \\
\end{array}$} & \multicolumn{2}{|c|}{$\begin{array}{c}\text { Pre-Delta* (Mar 2020-Jun 2021) } \\
n=1,076,745\end{array}$} & \multicolumn{2}{|c|}{$\begin{array}{c}\text { Delta* (Jul-Sep 2021) } \\
\mathrm{n}=172,889\end{array}$} \\
\hline & $\begin{array}{c}\text { Total } \\
\mathrm{N}=1,249,634\end{array}$ & $\begin{array}{l}\text { No COVID-19 } \\
n=1,227,981\end{array}$ & $\begin{array}{l}\text { COVID-19 } \\
n=21,653\end{array}$ & $\begin{array}{l}\text { No COVID-19 } \\
n=1,058,651\end{array}$ & $\begin{array}{l}\text { COVID-19 } \\
n=18,094\end{array}$ & $\begin{array}{c}\text { No COVID-19 } \\
n=169,330\end{array}$ & $\begin{array}{l}\text { COVID }-19 \\
\mathrm{n}=3,559\end{array}$ \\
\hline $\begin{array}{l}\text { Maternal age, } \\
\text { median (SD) }\end{array}$ & $29.0(5.8)$ & $29.0(5.8)$ & $28.0(6.0)$ & $29.0(5.8)$ & $28.0(6.0)$ & $29.0(5.7)$ & $28.0(5.8)$ \\
\hline \multicolumn{8}{|c|}{ Maternal race/ethnicity } \\
\hline White, non-Hispanic & $671,392(53.7)$ & $663,136(54.0)$ & $8,256(38.1)$ & $574,368(54.3)$ & $6,660(36.8)$ & $88,768(52.4)$ & $1,596(44.8)$ \\
\hline Hispanic & $230,836(18.5)$ & $223,784(18.2)$ & $7,052(32.6)$ & $188,114(17.8)$ & $6,164(34.1)$ & $35,670(21.1)$ & $888(25.0)$ \\
\hline Black, non-Hispanic & $181,143(14.5)$ & $177,508(14.5)$ & $3,635(16.8)$ & $153,408(14.5)$ & $2,947(16.3)$ & $24,100(14.2)$ & $688(19.3)$ \\
\hline Asian & $57,535(4.6)$ & $56,855(4.6)$ & $680(3.1)$ & $49,583(4.7)$ & $604(3.3)$ & $7,272(4.3)$ & $76(2.1)$ \\
\hline $\begin{array}{l}\text { Other/Unknown, } \\
\text { non-Hispanic }\end{array}$ & $108,728(8.7)$ & $106,698(8.7)$ & $2,030(9.4)$ & $93,178(8.8)$ & $1,719(9.5)$ & $13,520(8.0)$ & $311(8.7)$ \\
\hline \multicolumn{8}{|l|}{ Primary payor } \\
\hline Private & $631,894(50.6)$ & $624,069(50.8)$ & $7,825(36.1)$ & $537,957(50.8)$ & $6,367(35.2)$ & $86,112(50.9)$ & $1,458(41.0)$ \\
\hline Medicaid & $534,139(42.7)$ & $521,739(42.5)$ & $12,400(57.3)$ & $450,813(42.6)$ & $10,548(58.3)$ & $70,926(41.9)$ & $1,852(52.0)$ \\
\hline Self-pay & $21,022(1.7)$ & $20,557(1.7)$ & $465(2.1)$ & $17,351(1.6)$ & $386(2.1)$ & $3,206(1.9)$ & $79(2.2)$ \\
\hline Other & $62,579(5.0)$ & $61,616(5.0)$ & $963(4.4)$ & $52,530(5.0)$ & $793(4.4)$ & $9,086(5.4)$ & $170(4.8)$ \\
\hline \multicolumn{8}{|l|}{ Hospital location } \\
\hline Rural & $159,634(12.8)$ & $157,006(12.8)$ & $2,628(12.1)$ & $134,615(12.7)$ & $2,014(11.1)$ & $22,391(13.2)$ & $614(17.3)$ \\
\hline Urban & $1,090,000(87.2)$ & $1,070,975(87.2)$ & $19,025(87.9)$ & $924,036(87.3)$ & $16,080(88.9)$ & $146,939(86.8)$ & $2,945(82.7)$ \\
\hline \multicolumn{8}{|c|}{ U.S. Census division } \\
\hline East North Central & $200,701(16.1)$ & $198,061(16.1)$ & $2,640(12.2)$ & $169,631(16.0)$ & $2,259(12.5)$ & $28,430(16.8)$ & $381(10.7)$ \\
\hline East South Central & $94,224(7.5)$ & $92,902(7.6)$ & $1,322(6.1)$ & $80,335(7.6)$ & $1,018(5.6)$ & $12,567(7.4)$ & 304 (8.5) \\
\hline Middle Atlantic & $147,774(11.8)$ & $144,423(11.8)$ & $3,351(15.5)$ & $124,755(11.8)$ & $3,123(17.3)$ & $19,668(11.6)$ & $228(6.4)$ \\
\hline Mountain & $91,554(7.3)$ & $90,458(7.4)$ & $1,096(5.1)$ & $77,393(7.3)$ & $939(5.2)$ & $13,065(7.7)$ & $157(4.4)$ \\
\hline New England & $25,158(2.0)$ & $24,892(2.0)$ & $266(1.2)$ & $21,463(2.0)$ & $246(1.4)$ & $3,429(2.0)$ & $20(0.6)$ \\
\hline Pacific & $126,615(10.1)$ & $124,277(10.1)$ & $2,338(10.8)$ & $107,760(10.2)$ & $1,890(10.4)$ & $16,517(9.8)$ & 448 (12.6) \\
\hline South Atlantic & $332,317(26.6)$ & $326,419(26.6)$ & $5,898(27.2)$ & $283,595(26.8)$ & $4,683(25.9)$ & $42,824(25.3)$ & $1,215(34.1)$ \\
\hline West North Central & $80,263(6.4)$ & $78,710(6.4)$ & $1,553(7.2)$ & $66,326(6.3)$ & $1,310(7.2)$ & $12,384(7.3)$ & $243(6.8)$ \\
\hline West South Central & $151,028(12.1)$ & $147,839(12.0)$ & $3,189(14.7)$ & $127,393(12.0)$ & $2,626(14.5)$ & $20,446(12.1)$ & $563(15.8)$ \\
\hline \multicolumn{8}{|l|}{ Obesity } \\
\hline No & $1,057,646(84.6)$ & $1,039,849(84.7)$ & $17,797(82.2)$ & $897,069(84.7)$ & $14,881(82.2)$ & $142,780(84.3)$ & $2,916(81.9)$ \\
\hline Yes & $191,988(15.4)$ & $188,132(15.3)$ & $3,856(17.8)$ & $161,582(15.3)$ & $3,213(17.8)$ & $26,550(15.7)$ & $643(18.1)$ \\
\hline \multicolumn{8}{|l|}{ Diabetes (any) ${ }^{\dagger}$} \\
\hline No & $1,109,053(88.8)$ & $1,090,087(88.8)$ & $18,966(87.6)$ & $940,575(88.8)$ & $15,803(87.3)$ & $149,512(88.3)$ & $3,163(88.9)$ \\
\hline Yes & $140,581(11.2)$ & $137,894(11.2)$ & $2,687(12.4)$ & $118,076(11.2)$ & 2,291 (12.7) & $19,818(11.7)$ & $396(11.1)$ \\
\hline \multicolumn{8}{|c|}{ Hypertensive disorders of pregnancy (any) ${ }^{\S}$} \\
\hline No & $1,034,519(82.8)$ & $1,016,918(82.8)$ & $17,601(81.3)$ & $877,063(82.8)$ & $14,678(81.1)$ & $139,855(82.6)$ & $2,923(82.1)$ \\
\hline Yes & $215,115(17.2)$ & $211,063(17.2)$ & $4,052(18.7)$ & $181,588(17.2)$ & $3,416(18.9)$ & $29,475(17.4)$ & $636(17.9)$ \\
\hline \multicolumn{8}{|c|}{ Multiple-gestation pregnancy } \\
\hline No & $1,226,534(98.2)$ & $1,205,299(98.2)$ & $21,235(98.1)$ & $1,039,095(98.2)$ & $17,751(98.1)$ & $166,204(98.2)$ & $3,484(97.9)$ \\
\hline Yes & $23,100(1.8)$ & $22,682(1.8)$ & $418(1.9)$ & $19,556(1.8)$ & $343(1.9)$ & $3,126(1.8)$ & $75(2.1)$ \\
\hline \multicolumn{8}{|l|}{ Smoking } \\
\hline No & $1,187,831(95.1)$ & $1,166,855(95.0)$ & $20,976(96.9)$ & $1,005,234(95.0)$ & $17,598(97.3)$ & $161,621(95.4)$ & $3,378(94.9)$ \\
\hline Yes & $61,803(4.9)$ & $61,126(5.0)$ & $677(3.1)$ & $53,417(5.0)$ & $496(2.7)$ & $7,709(4.6)$ & $181(5.1)$ \\
\hline \multicolumn{8}{|l|}{ Stillbirth } \\
\hline No & $1,241,480(99.3)$ & $1,220,100(99.4)$ & $21,380(98.7)$ & $1,051,845(99.4)$ & $17,917(99.0)$ & $168,255(99.4)$ & $3,463(97.3)$ \\
\hline Yes & $8,154(0.7)$ & $7,881(0.6)$ & $273(1.3)$ & $6,806(0.6)$ & $177(1.0)$ & $1,075(0.6)$ & $96(2.7)$ \\
\hline \multicolumn{8}{|c|}{ Timing of stillbirth, wks (trimester)** } \\
\hline $20-27$ (2nd) & $3,607(44.2)$ & $3,498(44.4)$ & 109 (39.9) & $3,058(44.9)$ & $77(43.5)$ & $440(40.9)$ & $32(33.3)$ \\
\hline $28-42(3 r d)$ & $4,547(55.8)$ & $4,383(55.6)$ & $164(60.1)$ & $3,748(55.1)$ & $100(56.5)$ & $635(59.1)$ & $64(66.7)$ \\
\hline $\begin{array}{l}\text { Gestational age at } \\
\text { stillbirth, wks, } \\
\text { median (SD) }\end{array}$ & $29.0(6.8)$ & $29.0(6.8)$ & $29.0(6.2)$ & $29.0(6.8)$ & $29.0(6.5)$ & $30.0(6.7)$ & 30.0 \\
\hline \multicolumn{8}{|c|}{ 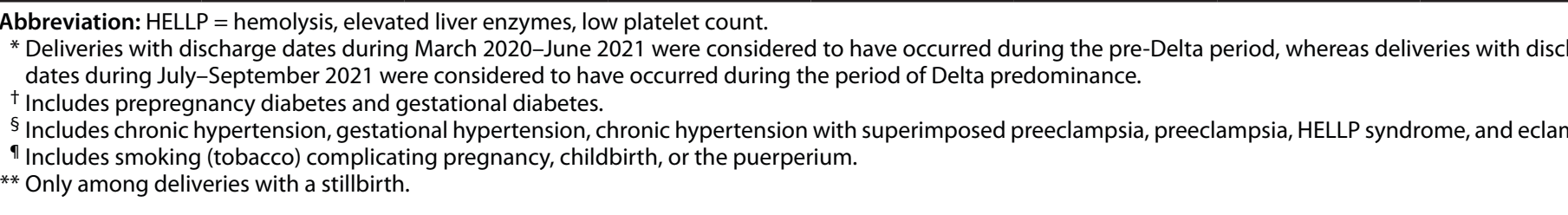 } \\
\hline
\end{tabular}


FIGURE. Relative risk for stillbirth among women with COVID-19 at delivery hospitalization compared with those without COVID-19 at delivery hospitalization — Premier Healthcare Database Special COVID-19 Release, United States, March 2020-September 2021*,t,\$

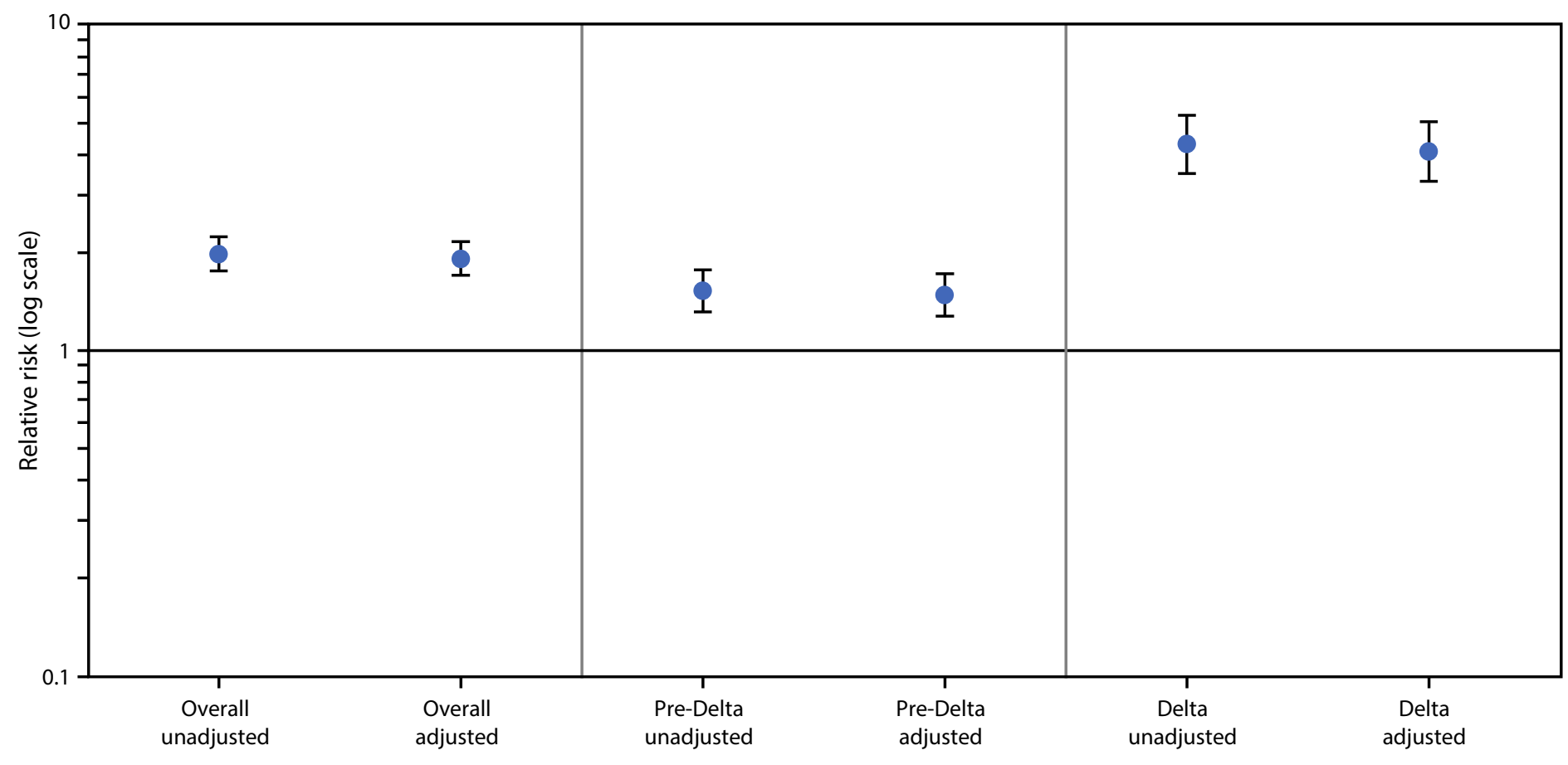

Abbreviation: $\mathrm{RR}=$ relative risk.

Period of variant predominance

* Deliveries with discharge dates during March 2020-June 2021 were considered to have occurred during the period preceding SARS-CoV-2 B.1.617.2 (Delta) variant predominance, whereas those with discharge dates during July-September 2021 were considered to have occurred during the period of Delta predominance.

† Overall: unadjusted $\mathrm{RR}=1.96(95 \% \mathrm{Cl}=1.74-2.21)$; adjusted $\mathrm{RR}=1.90(95 \% \mathrm{Cl}=1.69-2.15)$; pre-Delta: unadjusted $\mathrm{RR}=1.52(95 \% \mathrm{Cl}=1.31-1.77)$; adjusted $\mathrm{RR}=1.47$ $(95 \% \mathrm{Cl}=1.27-1.71)$; Delta: unadjusted $\mathrm{RR}=4.25(95 \% \mathrm{Cl}=3.46-5.22)$; adjusted $\mathrm{RR}=4.04(95 \% \mathrm{Cl}=3.28-4.97)$; $\mathrm{p}$-value for effect modification by period (pre-Delta period versus period of Delta predominance): $<0.001$.

$\S$ Models accounted for within-facility and within-woman correlation, and were adjusted for maternal age, race/ethnicity (Hispanic, non-Hispanic Black, non-Hispanic White, and non-Hispanic other), primary payor (Medicaid, private insurance, and other), obesity, smoking, any diabetes, any hypertension, and multiple-gestation pregnancy.

The rates of stillbirth in women without COVID-19 at delivery in this analysis $(0.64 \%$ overall $)$ were similar to the known prepandemic stillbirth rate of $0.59 \%$ (6). However, $0.98 \%$ of COVID-19-affected deliveries pre-Delta and $2.70 \%$ during the Delta period resulted in stillbirth. Data on the association between COVID-19 in pregnancy and stillbirth are emerging. Two metaanalyses found an association between COVID-19 during pregnancy and stillbirth but were unable to adjust for potential confounders $(2,4)$. In a previous analysis of the PHD-SR data, comparing women with and without COVID-19 documented at the delivery hospitalization during March-September 2020, the risk for stillbirth was not significantly increased after adjusting for confounders (3). The current analysis includes an additional year of data, adding to the growing evidence that COVID-19 is associated with an increased risk for stillbirth.

Delta became the predominant variant of SARS-CoV-2 in the United States in July 2021. ${ }^{\$ \$ \$ \$}$ The Delta variant is more

\footnotetext{
\$S\$S https://www.reuters.com/world/us/delta-variant-already-dominant-us-cdc-
} estimates-show-2021-07-07/ infectious and is associated with increased risk for hospitalization compared with previous variants $(7,8)$; however, nonpregnant patients are not more likely to have severe outcomes during hospitalization (9). In this analysis, the association between COVID-19 and stillbirth was stronger during the period of Delta predominance. Further studies that examine the effect of SARS-CoV-2 infection, including with the Delta variant, on fetal well-being are warranted.

The findings in this report are subject to at least seven limitations. First, the analysis relied on administrative data from hospital discharge ICD-10-CM codes; thus, identification of COVID-19 status, underlying medical conditions, gestational age, and stillbirths might be misclassified. Second, gestational age at SARS-CoV-2 infection was not available, and it is unknown whether COVID-19 diagnoses documented during the delivery hospitalization represented current or past infection. Third, many hospitals implemented universal SARS-CoV-2 testing among pregnant women assessed in labor and delivery units during spring 2020 (10), which would increase the detection of asymptomatic COVID-19. Laboratory information was unavailable for most hospitals in 
TABLE 2. Risk for stillbirth by maternal health characteristics and indicators of severe illness among delivery hospitalizations with a documented COVID-19 diagnosis — Premier Healthcare Database Special COVID-19 Release, United States, March 2020-September 2021

\begin{tabular}{|c|c|c|c|c|c|c|c|c|c|c|c|c|c|}
\hline \multirow[b]{3}{*}{ Characteristic } & \multicolumn{4}{|c|}{$\begin{array}{c}\text { Overall } \\
\mathrm{N}=21,653\end{array}$} & \multicolumn{4}{|c|}{$\begin{array}{l}\text { Pre-Delta* (Mar 2020-Jun 2021) } \\
\qquad \mathrm{n}=18,094\end{array}$} & \multicolumn{4}{|c|}{$\begin{array}{l}\text { Delta* (Jul-Sep 2021) } \\
\quad \mathrm{n}=3,559\end{array}$} & \multirow[b]{3}{*}{$p$-value } \\
\hline & \multicolumn{2}{|c|}{$\begin{array}{l}\text { Outcome } \\
\text { No. }(\%)\end{array}$} & \multicolumn{2}{|c|}{$\begin{array}{c}\mathrm{RR} \\
(95 \% \mathrm{Cl}) \\
\end{array}$} & \multicolumn{2}{|c|}{$\begin{array}{l}\text { Outcome } \\
\text { No. }(\%)\end{array}$} & \multicolumn{2}{|c|}{$\begin{array}{c}\text { RR } \\
(95 \% \mathrm{Cl}) \\
\end{array}$} & \multicolumn{2}{|c|}{$\begin{array}{l}\text { Outcome } \\
\text { No. }(\%)\end{array}$} & \multicolumn{2}{|c|}{$\begin{array}{c}\mathrm{RR} \\
(95 \% \mathrm{Cl}) \\
\end{array}$} & \\
\hline & $\begin{array}{l}\text { No } \\
\text { stillbirth }\end{array}$ & Stillbirth & Unadjusted & Adjusted $^{\dagger}$ & $\begin{array}{l}\text { No } \\
\text { stillbirth }\end{array}$ & Stillbirth & Unadjusted & Adjusted $^{\dagger}$ & $\begin{array}{l}\text { No } \\
\text { stillbirth }\end{array}$ & Stillbirth & Unadjusted & Adjusted $^{\dagger}$ & \\
\hline $\begin{array}{l}\text { Hypertensive } \\
\text { disorders of } \\
\text { pregnancy } \\
\text { (any) }\end{array}$ & $\begin{array}{l}3,995 \\
(18.7)\end{array}$ & $\begin{array}{c}57 \\
(20.9)\end{array}$ & $\begin{array}{c}1.15 \\
(0.86-1.53)\end{array}$ & $\begin{array}{c}1.08 \\
(0.81-1.44)\end{array}$ & $\begin{array}{l}3,379 \\
(18.9)\end{array}$ & $\begin{array}{c}37 \\
(20.9)\end{array}$ & $\begin{array}{c}1.14 \\
(0.79-1.63)\end{array}$ & $\begin{array}{c}1.05 \\
(0.73-1.50)\end{array}$ & $\begin{array}{c}616 \\
(17.8)\end{array}$ & $\begin{array}{c}20 \\
(20.8)\end{array}$ & $\begin{array}{c}1.21 \\
(0.74-1.96)\end{array}$ & $\begin{array}{c}1.19 \\
(0.74-1.92)\end{array}$ & $<0.001$ \\
\hline $\begin{array}{l}\text { Chronic } \\
\text { hypertension }\end{array}$ & $\begin{array}{l}515 \\
(2.4)\end{array}$ & $\begin{array}{c}13 \\
(4.8)\end{array}$ & $\begin{array}{c}2.00 \\
(1.15-3.47)\end{array}$ & $\begin{array}{c}1.79 \\
(1.03-3.11)\end{array}$ & $\begin{array}{l}418 \\
(2.3)\end{array}$ & $\begin{array}{c}7 \\
(4.0)\end{array}$ & $\begin{array}{c}1.71 \\
(0.81-3.62)\end{array}$ & $\begin{array}{c}1.49 \\
(0.70-3.19)\end{array}$ & $\begin{array}{c}97 \\
(2.8)\end{array}$ & $\begin{array}{c}6 \\
(6.3)\end{array}$ & $\begin{array}{c}2.24 \\
(1.00-4.99)\end{array}$ & $\begin{array}{c}2.11 \\
(0.94-4.74)\end{array}$ & 0.02 \\
\hline $\begin{array}{l}\text { Pregnancy- } \\
\text { associated } \\
\text { hypertension** }\end{array}$ & $\begin{array}{l}3,480 \\
(16.3)\end{array}$ & $\begin{array}{c}44 \\
(16.1)\end{array}$ & $\begin{array}{c}0.99 \\
(0.72-1.36)\end{array}$ & $\begin{array}{c}0.94 \\
(0.68-1.29)\end{array}$ & $\begin{array}{l}2,961 \\
(16.5)\end{array}$ & $\begin{array}{c}30 \\
(16.9)\end{array}$ & $\begin{array}{c}1.03 \\
(0.70-1.52)\end{array}$ & $\begin{array}{c}0.97 \\
(0.66-1.43)\end{array}$ & $\begin{array}{c}519 \\
(15.0)\end{array}$ & $\begin{array}{c}14 \\
(14.6)\end{array}$ & $\begin{array}{c}0.97 \\
(0.66-1.43)\end{array}$ & $\begin{array}{c}0.96 \\
(0.55-1.69)\end{array}$ & 0.005 \\
\hline Obesity & $\begin{array}{l}3,810 \\
(17.8)\end{array}$ & $\begin{array}{c}46 \\
(16.8)\end{array}$ & $\begin{array}{c}0.94 \\
(0.68-1.28)\end{array}$ & $\begin{array}{c}0.90 \\
(0.66-1.23)\end{array}$ & $\begin{array}{l}3,181 \\
(17.8)\end{array}$ & $\begin{array}{c}32 \\
(18.1)\end{array}$ & $\begin{array}{c}1.02 \\
(0.70-1.50)\end{array}$ & $\begin{array}{c}0.97 \\
(0.66-1.42)\end{array}$ & $\begin{array}{c}629 \\
(18.2)\end{array}$ & $\begin{array}{c}14 \\
(14.6)\end{array}$ & $\begin{array}{c}0.77 \\
(0.44-1.36)\end{array}$ & $\begin{array}{c}0.78 \\
(0.44-1.37)\end{array}$ & 0.02 \\
\hline $\begin{array}{c}\text { Diabetes } \\
(\text { any })^{\dagger+}\end{array}$ & $\begin{array}{l}2,659 \\
(12.4)\end{array}$ & $\begin{array}{c}28 \\
(10.3)\end{array}$ & $\begin{array}{c}0.81 \\
(0.55-1.19)\end{array}$ & $\begin{array}{c}0.80 \\
(0.53-1.18)\end{array}$ & $\begin{array}{l}2,273 \\
(12.7)\end{array}$ & $\begin{array}{c}18 \\
(10.2)\end{array}$ & $\begin{array}{c}0.78 \\
(0.48-1.27)\end{array}$ & $\begin{array}{c}0.78 \\
(0.47-1.30)\end{array}$ & $\begin{array}{c}386 \\
(11.1)\end{array}$ & $\begin{array}{c}10 \\
(10.4)\end{array}$ & $\begin{array}{c}0.93 \\
(0.49-1.77)\end{array}$ & $\begin{array}{c}0.88 \\
(0.46-1.67)\end{array}$ & 0.005 \\
\hline Smoking ${ }^{\S \S}$ & $\begin{array}{l}663 \\
(3.1)\end{array}$ & $\begin{array}{c}14 \\
(5.1)\end{array}$ & $\begin{array}{c}1.67 \\
(0.98-2.85)\end{array}$ & $\begin{array}{c}1.56 \\
(0.91-2.68)\end{array}$ & $\begin{array}{l}488 \\
(2.7)\end{array}$ & $\begin{array}{c}8 \\
(4.5)\end{array}$ & $\begin{array}{c}1.68 \\
(0.83-3.39)\end{array}$ & $\begin{array}{c}1.60 \\
(0.79-3.27)\end{array}$ & $\begin{array}{l}175 \\
(5.1)\end{array}$ & $\begin{array}{c}6 \\
(6.3)\end{array}$ & $\begin{array}{c}1.24 \\
(0.55-2.80)\end{array}$ & $\begin{array}{c}1.09 \\
(0.47-2.52)\end{array}$ & 0.18 \\
\hline $\begin{array}{l}\text { Multiple- } \\
\text { gestation } \\
\text { pregnancy }\end{array}$ & $\begin{array}{l}399 \\
(1.9)\end{array}$ & $\begin{array}{c}19 \\
(7.0)\end{array}$ & $\begin{array}{c}3.80 \\
(2.41-6.00)\end{array}$ & $\begin{array}{c}3.54 \\
(2.24-5.59)\end{array}$ & $\begin{array}{l}330 \\
(1.8)\end{array}$ & $\begin{array}{c}13 \\
(7.3)\end{array}$ & $\begin{array}{c}4.10 \\
(2.36-7.14)\end{array}$ & $\begin{array}{c}3.76 \\
(2.16-6.57)\end{array}$ & $\begin{array}{c}69 \\
(2.0)\end{array}$ & $\begin{array}{c}6 \\
(6.3)\end{array}$ & $\begin{array}{c}3.10 \\
(1.40-6.85)\end{array}$ & $\begin{array}{c}3.04 \\
(1.35-6.82)\end{array}$ & 0.11 \\
\hline $\begin{array}{l}\text { Adverse cardiac } \\
\text { event/ } \\
\text { outcome }\end{array}$ & $\begin{array}{l}160 \\
(0.7)\end{array}$ & $\begin{array}{c}10 \\
(3.7)\end{array}$ & $\begin{array}{c}4.81 \\
(2.60-8.87)\end{array}$ & $\begin{array}{c}4.44 \\
(2.38-8.29)\end{array}$ & $\begin{array}{l}120 \\
(0.7)\end{array}$ & $\begin{array}{c}4 \\
(2.3)\end{array}$ & $\begin{array}{c}3.35 \\
(1.26-8.89)\end{array}$ & $\begin{array}{c}3.09 \\
(1.15-8.34)\end{array}$ & $\begin{array}{c}40 \\
(1.2)\end{array}$ & $\begin{array}{c}6 \\
(6.3)\end{array}$ & $\begin{array}{c}5.09 \\
(2.35-11.03)\end{array}$ & $\begin{array}{c}5.18 \\
(2.34-11.48)\end{array}$ & 0.03 \\
\hline $\begin{array}{l}\text { Placental } \\
\text { abruption }\end{array}$ & $\begin{array}{l}273 \\
(1.3)\end{array}$ & $\begin{array}{c}36 \\
(13.2)\end{array}$ & $\begin{array}{c}10.49 \\
(7.53-14.63)\end{array}$ & $\begin{array}{c}10.12 \\
(7.28-14.08)\end{array}$ & $\begin{array}{l}206 \\
(1.1)\end{array}$ & $\begin{array}{c}22 \\
(12.4)\end{array}$ & $\begin{array}{c}11.12 \\
(7.26-17.05)\end{array}$ & $\begin{array}{c}10.63 \\
(6.96-16.22)\end{array}$ & $\begin{array}{c}67 \\
(1.9)\end{array}$ & $\begin{array}{c}14 \\
(14.6)\end{array}$ & $\begin{array}{c}7.33 \\
(4.35-12.36)\end{array}$ & $\begin{array}{c}7.53 \\
(4.47-12.66)\end{array}$ & 0.07 \\
\hline Sepsis & $\begin{array}{l}306 \\
(1.4)\end{array}$ & $\begin{array}{c}10 \\
(3.7)\end{array}$ & $\begin{array}{c}2.57 \\
(1.38-4.78)\end{array}$ & $\begin{array}{c}2.55 \\
(1.37-4.76)\end{array}$ & $\begin{array}{l}211 \\
(1.2)\end{array}$ & $\begin{array}{c}6 \\
(3.4)\end{array}$ & $\begin{array}{c}2.89 \\
(1.30-6.45)\end{array}$ & $\begin{array}{c}2.83 \\
(1.27-6.31)\end{array}$ & $\begin{array}{c}95 \\
(2.7)\end{array}$ & $\begin{array}{c}4 \\
(4.2)\end{array}$ & $\begin{array}{c}1.52 \\
(0.57-4.05)\end{array}$ & $\begin{array}{c}1.58 \\
(0.59-4.21)\end{array}$ & 0.56 \\
\hline Shock & $\begin{array}{l}121 \\
(0.6)\end{array}$ & $\begin{array}{c}15 \\
(5.5)\end{array}$ & $\begin{array}{c}9.20 \\
(5.62-15.05)\end{array}$ & $\begin{array}{c}9.31 \\
(5.65-15.35)\end{array}$ & $\begin{array}{c}91 \\
(0.5)\end{array}$ & $\begin{array}{c}8 \\
(4.5)\end{array}$ & $\begin{array}{c}8.60 \\
(4.35-17.00)\end{array}$ & $\begin{array}{c}8.70 \\
(4.35-17.39)\end{array}$ & $\begin{array}{l}30 \\
(0.9)\end{array}$ & $\begin{array}{c}7 \\
(7.3)\end{array}$ & $\begin{array}{c}7.49 \\
(3.73-15.04)\end{array}$ & $\begin{array}{c}7.95 \\
(3.95-16.00)\end{array}$ & 0.07 \\
\hline $\begin{array}{l}\text { Acute respiratory } \\
\text { distress } \\
\text { syndrome }\end{array}$ & $\begin{array}{l}915 \\
(4.3)\end{array}$ & $\begin{array}{c}25 \\
(9.2)\end{array}$ & $\begin{array}{c}2.22 \\
(1.48-3.33)\end{array}$ & $\begin{array}{c}2.16 \\
(1.44-3.23)\end{array}$ & $\begin{array}{l}601 \\
(3.4)\end{array}$ & $\begin{array}{c}12 \\
(6.8)\end{array}$ & $\begin{array}{c}2.07 \\
(1.16-3.71)\end{array}$ & $\begin{array}{c}2.01 \\
(1.13-3.59)\end{array}$ & $\begin{array}{l}314 \\
(9.1)\end{array}$ & $\begin{array}{c}13 \\
(13.5)\end{array}$ & $\begin{array}{c}1.55 \\
(0.87-2.75)\end{array}$ & $\begin{array}{c}1.53 \\
(0.87-2.70)\end{array}$ & 0.09 \\
\hline $\begin{array}{l}\text { Mechanical } \\
\text { ventilation }\end{array}$ & $\begin{array}{l}379 \\
(1.8)\end{array}$ & $\begin{array}{c}20 \\
(7.3)\end{array}$ & $\begin{array}{c}4.21 \\
(2.70-6.57)\end{array}$ & $\begin{array}{c}4.12 \\
(2.62-6.48)\end{array}$ & $\begin{array}{l}257 \\
(1.4)\end{array}$ & $\begin{array}{c}12 \\
(6.8)\end{array}$ & $\begin{array}{c}4.82 \\
(2.72-8.55)\end{array}$ & $\begin{array}{c}4.79 \\
(2.67-8.61)\end{array}$ & $\begin{array}{c}122 \\
(3.5)\end{array}$ & $\begin{array}{c}8 \\
(8.3)\end{array}$ & $\begin{array}{c}2.40 \\
(1.19-4.84)\end{array}$ & $\begin{array}{c}2.41 \\
(1.17-4.95)\end{array}$ & 0.57 \\
\hline ICU admission & $\begin{array}{c}1,074 \\
(5.0)\end{array}$ & $\begin{array}{c}36 \\
(13.2)\end{array}$ & $\begin{array}{c}2.81 \\
(1.99-3.97)\end{array}$ & $\begin{array}{c}2.74 \\
(1.93-3.89)\end{array}$ & $\begin{array}{l}800 \\
(4.5)\end{array}$ & $\begin{array}{c}18 \\
(10.2)\end{array}$ & $\begin{array}{c}2.39 \\
(1.48-3.87)\end{array}$ & $\begin{array}{c}2.31 \\
(1.42-3.76)\end{array}$ & $\begin{array}{l}274 \\
(7.9)\end{array}$ & $\begin{array}{c}18 \\
(18.8)\end{array}$ & $\begin{array}{c}2.58 \\
(1.57-4.25)\end{array}$ & $\begin{array}{c}2.57 \\
(1.54-4.28)\end{array}$ & 0.003 \\
\hline
\end{tabular}

Abbreviations: HELLP = hemolysis, elevated liver enzymes, low platelet count; ICU = intensive care unit; RR = relative risk.

* Deliveries with discharge dates during March 2020-June 2021 were considered to occur during the pre-Delta period, whereas deliveries with discharges dates during July-September 2021 were considered to occur during the period of Delta predominance.

${ }^{\dagger}$ Models accounted for within-facility and within-woman correlation, and were adjusted for maternal age, race/ethnicity (Hispanic, non-Hispanic Black, non-Hispanic White, and non-Hispanic other), and primary payor (Medicaid, private insurance, and other).

$\S$ Assessing for effect modification by period (pre-Delta versus period of Delta predominance), based on interaction term added to adjusted model.

"Includes chronic hypertension, gestational hypertension, chronic hypertension with superimposed preeclampsia, preeclampsia, HELLP syndrome, and eclampsia.

** Includes gestational hypertension, chronic hypertension with superimposed preeclampsia, preeclampsia, HELLP syndrome, and eclampsia.

${ }^{+\dagger}$ Includes prepregnancy diabetes and gestational diabetes.

$\S \S$ Includes smoking (tobacco) complicating pregnancy, childbirth, or the puerperium.

१ๆ Includes acute myocardial infarction, cardiomyopathy, heart failure/arrest during surgery or procedure, cardiac arrest/ventricular fibrillation, conversion of cardiac rhythm, incident ventricular tachycardia, ischemia, pulmonary edema/acute heart failure, and atrial fibrillation/atrial flutter/supraventricular tachycardia.

PHD-SR and therefore not used in this analysis; if participating hospitals had different screening practices, some patients with SARS-CoV-2 infection might have been missed or misclassified. In hospitals not conducting universal SARS-CoV-2 testing, women experiencing adverse outcomes during the delivery hospitalization, including stillbirth, might have been more likely to be tested for SARS-CoV-2 infection. Fourth, because outpatient records were not universally available, and linkage across different hospital systems was not possible, the analysis was restricted to codes included during the delivery hospitalization and did not examine COVID-19 diagnoses or underlying medical conditions recorded before the delivery hospitalization (i.e., during a prenatal visit). Fifth, whole genome sequencing data were not available to confirm the variant of SARS-CoV-2 for this analysis, and period was used as a proxy; however, the Delta variant accounted for $>90 \%$ of U.S. COVID-19 cases during July-September 2021.9999 Sixth, it was not

\footnotetext{
9999 https://covid.cdc.gov/covid-data-tracker/\#variant-proportions
} 


\section{Summary}

What is already known about this topic?

Pregnant women are at increased risk for severe disease from COVID-19, and COVID-19 is associated with an increased risk for adverse perinatal outcomes.

What is added by this report?

Among 1,249,634 delivery hospitalizations during March 2020-September 2021, U.S. women with COVID-19 were at increased risk for stillbirth compared with women without COVID-19 (adjusted relative risk [aRR] = 1.90; $95 \% \mathrm{Cl}=1.69-2.15)$. The magnitude of association was higher during the period of SARS-CoV-2 B.1.617.2 (Delta) variant predominance than during the pre-Delta period.

What are the implications for public health practice?

Implementing evidence-based COVID-19 prevention strategies, including vaccination before or during pregnancy, is critical to reduce the impact of COVID-19 on stillbirths.

possible to assess vaccination status in this analysis. However, because COVID-19 vaccines are highly effective, ${ }^{* * * * *}$ and COVID-19 vaccination coverage among pregnant women was approximately $30 \%$ as of July $2021,+{ }^{+\dagger \dagger \dagger}$ most women with COVID-19 at delivery were likely unvaccinated. Finally, although the PHD-SR included a large population across U.S. Census divisions, it represents delivery hospitalizations from a convenience sample of reporting hospitals, limiting generalizability of results to the U.S. population.

This analysis adds to growing evidence of an association between COVID-19 in pregnancy and stillbirth, highlights that the risk for stillbirth associated with COVID-19 is affected by maternal morbidity, and demonstrates that the risk has increased during the Delta period. Further investigation from prospective studies is warranted to confirm these findings, identify the biologic mechanism for the observed increased risk for stillbirth with maternal COVID-19, and assess differences in risks relative to the timing and severity of infection and the contribution of maternal risk factors. In addition, further investigation of vaccine effectiveness during pregnancy, including prevention of stillbirth, is warranted. Most importantly, these findings underscore the importance of COVID-19 prevention strategies, including vaccination before or during pregnancy.

\footnotetext{
***** https://covid.cdc.gov/covid-data-tracker/\#vaccine-effectiveness

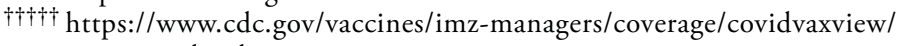
interactive.html
}

\section{Acknowledgments}

Tegan Boehmer, Lara Bull, Jennifer Wiltz, Data, Analytics, and Visualization Task Force, CDC COVID-19 Response Team; Romeo Galang, Suzanne Gilboa, Titilope Oduyebo, Emily O’Malley Olsen, Maria Rivera, Neha Shinde, Van Tong, Kate Woodworth, Lauren Zapata, Pregnancy and Infant Linked Outcomes Team, CDC COVID-19 Response Team.

Corresponding author: Carla L. DeSisto, eocevent397@cdc.gov.

${ }^{1}$ CDC COVID-19 Response Team.

All authors have completed and submitted the International Committee of Medical Journal Editors form for disclosure of potential conflicts of interest. No potential conflicts of interest were disclosed.

\section{References}

1. Zambrano LD, Ellington S, Strid P, et al.; CDC COVID-19 Response Pregnancy and Infant Linked Outcomes Team. Update: characteristics of symptomatic women of reproductive age with laboratory-confirmed SARS-CoV-2 infection by pregnancy status - United States, January 22 October 3, 2020. MMWR Morb Mortal Wkly Rep 2020;69:1641-7. PMID:33151921 https://doi.org/10.15585/mmwr.mm6944e3

2. Allotey J, Stallings E, Bonet M, et al.; for PregCOV-19 Living Systematic Review Consortium. Clinical manifestations, risk factors, and maternal and perinatal outcomes of coronavirus disease 2019 in pregnancy: living systematic review and meta-analysis. BMJ 2020;370:m3320. PMID:32873575 https://doi.org/10.1136/bmj.m3320

3. Ko JY, DeSisto CL, Simeone RM, et al. Adverse pregnancy outcomes, maternal complications, and severe illness among US delivery hospitalizations with and without a coronavirus disease 2019 (COVID-19) diagnosis. Clin Infect Dis 2021;73(Suppl 1):S24-31. PMID:33977298 https://doi.org/10.1093/cid/ciab344

4. Wei SQ, Bilodeau-Bertrand M, Liu S, Auger N. The impact of COVID-19 on pregnancy outcomes: a systematic review and metaanalysis. CMAJ 2021;193:E540-8. PMID:33741725 https://doi. org/10.1503/cmaj.202604

5. Di Girolamo RD, Khalil A, Alameddine S, et al. Placental histopathology after SARS-CoV-2 infection in pregnancy: a systematic review and meta-analysis. Am J Obstet Gynecol MFM 2021;3:100468. PMID:34425296 https://doi.org/10.1016/j.ajogmf.2021.100468

6. Hoyert DL, Gregory ECW. Cause-of-death data from the fetal death file, 2015-2017. Natl Vital Stat Rep 2020;69:1-20. PMID:32510316

7. Allen H, Vusirikala A, Flannagan J, et al.; COVID-19 Genomics UK (COG-UK Consortium). Household transmission of COVID-19 cases associated with SARS-CoV-2 delta variant (B.1.617.2): national casecontrol study. Lancet Reg Health Eur 2021;100252. PMID:34729548 https://doi.org/10.1016/j.lanepe.2021.100252

8. Sheikh A, McMenamin J, Taylor B, Robertson C; Public Health Scotland and the EAVE II Collaborators. SARS-CoV-2 Delta VOC in Scotland: demographics, risk of hospital admission, and vaccine effectiveness. Lancet 2021;397:2461-2. PMID:34139198 https://doi.org/10.1016/ S0140-6736(21)01358-1

9. Taylor CA, Patel K, Pham H, et al.; COVID-NET Surveillance Team. Severity of disease among adults hospitalized with laboratory-confirmed COVID-19 before and during the period of SARS-CoV-2 B.1.617.2 (Delta) predominance-COVID-NET, 14 states, January-August 2021. MMWR Morb Mortal Wkly Rep 2021;70:1513-9. PMID:34710076 https://doi.org/10.15585/mmwr.mm7043e1

10. Adhikari EH, Moreno W, Zofkie AC, et al. Pregnancy outcomes among women with and without severe acute respiratory syndrome coronavirus 2 infection. JAMA Netw Open 2020;3:e2029256. PMID:33211113 https://doi.org/10.1001/jamanetworkopen.2020.29256 